\title{
Golden Ratio Phenomenon of Random Data Obeying von Karman Spectrum
}

\author{
Ming $\mathrm{Li}^{1}$ and Wei Zhao ${ }^{2}$ \\ ${ }^{1}$ School of Information Science \& Technology, East China Normal University, No. 500, Dong-Chuan Road, Shanghai 200241, China \\ ${ }^{2}$ Department of Computer and Information Science, University of Macau, Avenue Padre Tomas Pereira, Taipa 1356, Macau \\ Correspondence should be addressed to Ming Li; ming_lihk@yahoo.com
}

Received 9 July 2013; Accepted 26 July 2013

Academic Editor: Ezzat G. Bakhoum

Copyright (c) 2013 M. Li and W. Zhao. This is an open access article distributed under the Creative Commons Attribution License, which permits unrestricted use, distribution, and reproduction in any medium, provided the original work is properly cited.

\begin{abstract}
von Karman originally deduced his spectrum of wind speed fluctuation based on the Stokes-Navier equation. Taking into account, the practical issues of measurement and/or computation errors, we suggest that the spectrum can be described from the point of view of the golden ratio. We call it the golden ratio phenomenon of the von Karman spectrum. To depict that phenomenon, we derive the von Karman spectrum based on fractional differential equations, which bridges the golden ratio to the von Karman spectrum and consequently provides a new outlook of random data following the von Karman spectrum in turbulence. In addition, we express the fractal dimension, which is a measure of local self-similarity, using the golden ratio, of random data governed by the von Karman spectrum.
\end{abstract}

\section{Instruction}

The golden ratio, denoted by $\varphi$, is an irrational number given by $\varphi=(1+\sqrt{5}) / 2$ [1]. The paper by Ackermann [2] may likely be the earliest literature on the golden ratio in a mathematics journal in English in 1895, but it attracted and has attracted the interest of scientists and engineers in various fields of sciences and engineering, ranging from chemistry to computer science; see, for example, [1], Benassi [3], Putz [4], Orita et al. [5], Perez [6], Hassaballah et al. [7], Kellerhals [8], Henein et al. [9], Hurtley [10], Coldea et al. [11], Affleck [12], Jones et al. [13], Kaygn et al. [14], Cervantes et al. [15], Chebotarev [16], Benavoli et al. [17], Manikantan et al. [18], Assimakis et al. [19], Good [20], Davis and Jahnke [21], Totland [22], Moufarrège [23], Boeyens [24], Iñiguez et al. [25], Andrews and Zhang [26], Hofri and Rosberg [27], Itai and Rosberg [28], Cassandras and Julka [29], and Tanackov et al. [30], just to mention a few.

In the field of random functions, more precisely, turbulence in fluid mechanics, a kind of power spectra density (PSD) function introduced by von Karman [31], known as the von Karman spectra (VKS), has been widely used in the diverse fields, ranging from turbulence to acoustic wave propagation in random media; see, for example, Goedecke et al. [32] and the references therein. Among the von Karman spectra, the spectrum (VKSW for short) expressed in (1) is particularly useful in the field of wind engineering for the modeling of wind speed fluctuation; see, for example, [33-41]. That PSD is in the form

$$
S_{\mathrm{von}}(f)=\frac{4 u_{f}^{2} b_{v} w}{f\left(1+70.8 w^{2}\right)^{5 / 6}}, \quad w=\frac{f L_{u}^{x}}{U}
$$

where $f$ is frequency $(\mathrm{Hz}), L_{u}^{x}$ is turbulence integral scale, $U$ is mean speed, $u_{f}$ is friction velocity $\left(\mathrm{ms}^{-1}\right)$, and $b_{v}$ is friction velocity coefficient such that the variance of wind speed $\sigma_{u}^{2}=$ $b_{v} u_{f}^{2}$.

Note that (1) was conventionally deduced based on the Stokes-Navier equation ([31], Bauer and Zeibig [42], Tropea [43], Monin and Yaglom [44], Xiushu [45]). It does not originally relate to the concept of either the golden ratio or fractal dimension. As a matter of fact, reports regarding turbulence's fractal dimension derived directly based on the Stokes-Navier equation are rarely seen as Gaoan stated in [46, page 55], letting alone the golden ratio. 
This paper aims at contributing the following three results. First, we will propose a rigorous but concise derivation of (1). Then, we will generalize (1) such that the generalization may be described from the point of view of the golden ratio. Finally, we will explain the golden ratio phenomenon of the VKSW from the point of view of fractal dimension or local self-similarity. As a result, we achieve the goal of bridging the golden ratio to the VKSW as well as selfsimilarity of random data, establishing a new outlook of data following the VKSW.

The rest of the paper is organized as follows. The preliminaries are briefed in Section 2. The results are given in Section 3, which is followed by conclusions.

\section{Preliminaries}

2.1. Golden Ratio. One of the conventional ways to deduce the golden ratio $\varphi$ is to solve the difference equation that produces the Fibonacci sequences. The equation is given by (see, e.g., [1], Jamieson [46], Ranum [47], and Eggar [48])

$$
F(n)=F(n-1)+F(n-2),
$$

$n \in \mathbf{N}$ (the set of natural numbers).

Denote by $Z_{F}(z)$ the $z$-transform of $F(n)$. Then, doing the $z$ transform on both sides of (2) yields

$$
Z_{F}(z)=Z_{F}(z)\left(z^{-1}+z^{-2}\right)
$$

The above expression can be rewritten as

$$
z^{2}-z-1=0
$$

The solutions to (4) are expressed by

$$
z_{1,2}=\left\{\begin{array}{l}
\frac{1+\sqrt{5}}{2} \\
\frac{1-\sqrt{5}}{2}
\end{array}\right.
$$

The golden ratio equals $z_{1}$; that is,

$$
\varphi=z_{1}=\frac{1+\sqrt{5}}{2} \approx 1.618
$$

In addition,

$$
z_{2}=\frac{1-\sqrt{5}}{2}=-\frac{1}{\varphi} \approx-0.618
$$

2.2. Fractional Oscillators. There are three types of fractional oscillators. The conventional type, see, for example, Achar et al. $[49,50]$, is given by

$$
\frac{d^{2-\varepsilon} x(t)}{d t^{2-\varepsilon}}+\omega_{0}^{2} x(t)=e(t), \quad 0<\varepsilon<1 .
$$

The second type was introduced by Lim and Muniandy [51]. It is in the form

$$
\left(\frac{d^{2}}{d t^{2}}+\lambda\right)^{\beta} x(t)=e(t), \quad \beta>0 .
$$

The third type introduced by Lim and Teo [52] is expressed by

$$
\left({ }_{a} D_{t}^{\alpha}+\lambda\right)^{\beta} x_{\alpha, \beta}(t)=e(t), \quad 0<\alpha<1, \beta>0 .
$$

The symbol ${ }_{a} D_{t}^{\alpha}$ is a fractional differential operator; see, for example, Eab and Lim [53, 54], Lim et al. [55], Klafter et al. [56], Machado et al. [57], and Cattani [58]. In what follows, we use (10) in the general sense.

We now consider the fractional differential equation with the coefficient $A$ in the form

$$
A\left(\frac{d}{d t}+\lambda\right)^{\beta} y_{\mathrm{fOU}}(t)=\eta(t), \quad \beta>0,
$$

where $\eta(t)$ is a white noise. Then, the solution to (11) is the fractional Ornstein-Uhlenbeck (OU) process [59], referring to Coffey et al. [60] for the meaning of OU process.

\section{Results}

Denote that $g_{\mathrm{fOU}}(t)$ is the impulse response function of (11). Then, it is the solution to the following equation with zero initial conditions

$$
A\left(\frac{d}{d t}+\lambda\right)^{\beta} g_{\mathrm{fOU}}(t)=\delta(t)
$$

where $\delta(t)$ is the Dirac- $\delta$ function. Doing the Fourier transforms on both sides of the above equation yields

$$
G_{\mathrm{fOU}}(f)=\frac{A}{(\lambda-j 2 \pi f)^{\beta}},
$$

where $G_{\mathrm{fOU}}(f)$ is the Fourier transform of $g_{\mathrm{fOU}}(t)$. by

Let $S_{y_{\mathrm{fOU}}}(f)$ be the PSD of $y_{\mathrm{fOU}}(t)$. Then, $S_{y_{\mathrm{fou}}}(f)$ is given

$$
S_{y_{\mathrm{fOU}}}(f)=\left|G_{\mathrm{fOU}}(f)\right|^{2}=\frac{A^{2}}{\left[\lambda^{2}+(2 \pi f)^{2}\right]^{\beta}} .
$$

Thus, we have the theorem below.

Theorem 1. Let $X_{v k}(t)$ be the random function that obeys (1). Then, $X_{v k}(t)$ is governed by the fractional differential equation that is in the form

$$
\sqrt{A_{v k}}\left(\frac{d}{d t}+B_{v k}\right)^{5 / 6} X_{v k}(t)=\eta(t) .
$$

Its solution in frequency domain is given by

$$
S_{v k}(f)=\frac{A_{v k}}{\left[\left(B_{v k}\right)^{2}+(2 \pi f)^{2}\right]^{5 / 6}} .
$$

Proof. Replacing $A, \lambda$, and $\beta$ in (11) with $\sqrt{A_{\mathrm{vk}}}, B_{\mathrm{vk}}$, and $5 / 6$, respectively, yields (15). Substituting $A, \lambda$, and $\beta$ in (14) with $\sqrt{A_{\mathrm{vk}}}, B_{\mathrm{vk}}$, and $5 / 6$, respectively, produces (16). Thus, Theorem 1 results.

From Theorem 1, we obtain the following corollary. 
Corollary 2 (modified VKSW). Let $X_{v k \varphi}(t)$ be the random function that is governed by the fractional differential equation given by

$$
\sqrt{A_{v k}}\left(\frac{d}{d t}+B_{v k}\right)^{\varphi / 2} X_{v k \varphi}(t)=\eta(t) .
$$

Then, its solution in frequency domain is in the form

$$
S_{v k \varphi}(f)=\frac{A_{v k}}{\left[\left(B_{v k}\right)^{2}+(2 \pi f)^{2}\right]^{\varphi / 2}} .
$$

The proof is straightforward and omitted consequently.

From Theorem 1 and Corollary 2, we immediately have the remark below.

Remark 3. The VKSW may be approximately expressed by the golden ratio.

As a matter of fact,

$$
\frac{\varphi}{2} \approx \frac{5}{6} .
$$

Thus, (16) approximately equals (18). This may not be a simple approximation but substantially develops the implication of the VKSW from the point of view of the golden ratio.

Note that there are errors in measuring real random data [61-63] and computation errors [64-66]. Thus, from a view of practice, the power of the VKSW may not exactly be the value of 5/6 in most cases in engineering. Rather, it may be in the form

$$
\left(\frac{5}{6}\right)+e
$$

where $e$ is error. Thus, by using the golden ratio, (18) is quite reasonable to characterize random functions that obey the VKSW.

For the purpose of exhibiting the results in time domain, we denote by $F$ and $F^{-1}$ the operator of the Fourier transform and its inverse, respectively. Then, we get the theorem below.

Theorem 4. The inverse Fourier transform of $1 /\left[1+(2 \pi f)^{2}\right]^{\varphi / 2}$ is given by

$$
\begin{aligned}
& F^{-1}\left\{\frac{1}{\left[1+(2 \pi f)^{2}\right]^{\varphi / 2}}\right\} \\
& =\frac{2 \sqrt{\pi}}{\Gamma(\varphi / 2)}\left(\frac{|\tau|}{2}\right)^{(\varphi-1) / 2} K_{(\varphi-1) / 2}(|\tau|),
\end{aligned}
$$

where $K_{v}(z)$ is the modified Bessel function of second kind or the MacDonald function and $\tau$ is the time lag.

Proof. Because $(\varphi-1) / 2>1 / 2$, according to the computation formula in Gelfand and Vilenkin [67, page 188, in Section 2, Chapter 2], (21) holds.
Recall that the Fourier transform of $|t|^{\alpha}$ is expressed by [68]

$$
F\left(|t|^{\alpha}\right)=-2 \sin \left(\frac{\alpha \pi}{2}\right) \Gamma(\alpha+1)|\omega|^{-\alpha-1},
$$

where $\alpha \neq 1,3, \ldots$

Note that

$$
S_{\mathrm{vk} \varphi}(f) \sim \frac{1}{f^{\varphi / 2}} \quad \text { for } f \longrightarrow \infty .
$$

Then, denoting $r_{\mathrm{vk} \varphi}(\tau)$ is the inverse Fourier transform of $S_{\mathrm{vk} \varphi}(f)$, one has

$$
r_{\mathrm{vk} \varphi}(\tau) \sim|\tau|^{(\varphi-1) / 2} \quad \text { for } \tau \longrightarrow 0 .
$$

The fractal dimension of a process can be determined by its autocorrelation function (ACF) for $\tau \rightarrow 0$ [69]. Thus,

$$
r_{\mathrm{vk}}(0)-r_{\mathrm{vk}}(\tau) \sim|\tau|^{(\varphi-1) / 2} \text { for } \tau \longrightarrow 0 .
$$

Therefore, with the probability one [69], the fractal dimension of the modified von Karman process based on the golden ratio is given by

$$
D_{\mathrm{vk} \varphi}=\left(2-\frac{\varphi-1}{4}\right)=\frac{7-\varphi}{4} .
$$

Approximately, it is expressed by

$$
D_{\mathrm{vk} \varphi} \approx 1.346 .
$$

Note that fractal dimension is a measure of local selfsimilarity, irregularity, or roughness [70]. High value of fractal dimension of a sample path implies high irregularity of that path. Thus, (26) means that the modified von Karman process with the golden ratio has considerable local irregularity.

We would like to call out the work described above as the golden ratio phenomenon of the von Karman process. From the point of view of our work in data science or big data, this research may not be enough. The future work will investigate possible golden ratio phenomena in other topics of data such as those discussed in [71-82], exploring laws associating with the golden ratio in the universe.

\section{Conclusions}

We have given the derivation of the von Karman spectrum based on the fractional differential equation (10). The results suggest that the process obeying VKSW is in the class of fractional OU processes. Moreover, we have explained the reasons why the VKSW may be described from the point of view of the golden ratio. The fractal dimension of random data obeying the VKSW by using the golden ratio has also been discussed.

\section{Acknowledgments}

This work was supported in part by the National Natural Science Foundation of China under the Project Grant nos. 61272402, 61070214, and 60873264 and by the 973 Plan under the Project Grant no. 2011CB302800. 


\section{References}

[1] M. Livio, The Golden Ration, Random House, Inc., 2003.

[2] E. C. Ackermann, "The golden section," The American Mathematical Monthly, vol. 2, no. 9-10, pp. 260-264, 1895.

[3] E. Benassi, "What do the azobenzene oligomer helixes have to do with the golden ratio?" Computational and Theoretical Chemistry, vol. 1014, pp. 13-23, 2013.

[4] M. V. Putz, "Valence atom with bohmian quantum potential: the golden ratio approach," Chemistry Central Journal, vol. 6, article 135, 2012.

[5] M. Orita, K. Ohno, and T. Niimi, "Two "golden ratio" indices in fragment-based drug discovery," Drug Discovery Today, vol. 14, no. 5-6, pp. 321-328, 2009.

[6] J. C. Perez, "Codon populations in single-stranded whole human genome DNA Are fractal and fine-tuned by the golden ratio 1.618," Interdisciplinary Sciences, Computational Life Sciences, vol. 2, no. 3, pp. 228-240, 2010.

[7] M. Hassaballah, K. Murakami, and S. Ido, "Face detection evaluation: a new approach based on the golden ratio $\Phi$," Signal, Image and Video Processing, vol. 7, no. 2, pp. 307-316, 2013.

[8] R. Kellerhals, "Scissors congruence, the golden ratio and volumes in hyperbolic 5-space," Discrete and Computational Geometry, vol. 47, no. 3, pp. 629-658, 2012.

[9] M. Y. Henein, Y. Zhao, R. Nicoll et al., "The human heart: application of the golden ratio and angle," International Journal of Cardiology, vol. 150, no. 3, pp. 239-242, 2011.

[10] S. Hurtley, "Hidden symmetry revealed," Science, vol. 327, no. 5962, p. 123, 2010.

[11] R. Coldea, D. A. Tennant, E. M. Wheeler et al., "Quantum criticality in an ising chain: experimental evidence for emergent e8 symmetry," Science, vol. 327, no. 5962, pp. 177-180, 2010.

[12] I. Affleck, "Solid-state physics: golden ratio seen in a magnet," Nature, vol. 464, no. 7287, pp. 362-363, 2010.

[13] P. W. Jones, M. Maggioni, and R. Schul, "Manifold parametrizations by eigenfunctions of the Laplacian and heat kernels," Proceedings of the National Academy of Sciences of the United States of America, vol. 105, no. 6, pp. 1803-1808, 2008.

[14] B. Kaygn, B. Balçin, C. Yildiz, and S. Arslan, "The effect of teaching the subject of Fibonacci numbers and golden ratio through the history of mathematics," Procedia-Social and Behavioral Sciences, vol. 15, pp. 961-965, 2011.

[15] E. Cervantes, J. J. Martín, R. Ardanuy, J. G. de Diego, and Á. Tocino, "Modeling the Arabidopsis seed shape by a cardioid: efficacy of the adjustment with a scale change with factor equal to the golden ratio and analysis of seed shape in ethylene mutants," Journal of Plant Physiology, vol. 167, no. 5, pp. 408410, 2010 .

[16] P. Chebotarev, "Spanning forests and the golden ratio," Discrete Applied Mathematics, vol. 156, no. 5, pp. 813-821, 2008.

[17] A. Benavoli, L. Chisci, and A. Farina, "Fibonacci sequence, golden section, Kalman filter and optimal control," Signal Processing, vol. 89, no. 8, pp. 1483-1488, 2009.

[18] K. Manikantan, B. V. Arun, and D. K. S. Yaradoni, "Optimal multilevel thresholds based on Tsallis entropy method using golden ratio particle swarm optimization for improved image segmentation," Procedia Engineering, vol. 30, pp. 364-371, 2012.

[19] N. Assimakis, M. Adam, and C. Triantafillou, "Lainiotis filter, golden section and Fibonacci sequence," Signal Processing, vol. 93, no. 4, pp. 721-730, 2013.
[20] I. J. Good, "Complex Fibonacci and Lucas numbers, continued fractions, and the square root of the golden ratio (condensed version)," The Journal of the Operational Research Society, vol. 43, no. 8, pp. 837-842, 1992.

[21] S. T. Davis and J. C. Jahnke, "Unity and the golden section: rules for aesthetic choice?" The American Journal of Psychology, vol. 104, no. 2, pp. 257-277, 1991.

[22] H. Totland, "Quartic polynomials and the golden ratio," Mathematics Magazine, vol. 82, no. 3, pp. 197-201, 2009.

[23] R. Moufarrège, "The golden ratios of the breast," in Breast Augmentation, Principles and Practice, chapter 13, Springer, New York, NY, USA, 2009.

[24] J. C. A. Boeyens, "Covalent interaction," in Electronic Structure and Number Theory, Structure and Bonding, J. C. A. Boeyens and P. Comba, Eds., vol. 148, Springer, New York, NY, USA, 2013.

[25] J. Iñiguez, A. Hansen, I. Pérez et al., "On division in extreme and mean ratio and its connection to a particular re-expression of the golden quadratic equation $x^{2}-x-1=0$," Nexus Network Journal, vol. 8, no. 2, pp. 93-100, 2006.

[26] M. Andrews and L. Zhang, "Creating templates to achieve low delay in multi-carrier frame-based wireless data systems," Wireless Networks, vol. 16, no. 6, pp. 1765-1776, 2010.

[27] M. Hofri and Z. Rosberg, "Packet delay under the golden ratio weighted TDM policy in a multiple-access channel," IEEE Transactions on Information Theory, vol. 33, no. 3, pp. 341-349, 1987.

[28] A. Itai and Z. Rosberg, "A golden ratio control policy for a multiple-access channel," IEEE Transactions on Automatic Control, vol. 29 , no. 8, pp. 712-718, 1984.

[29] C. G. Cassandras and V. Julka, "Scheduling policies using marked/phantom slot algorithms," Queueing Systems, vol. 20, no. 1-2, pp. 207-254, 1995.

[30] I. Tanackov, G. Stojić, J. Tepić, M. Kostelac, F. Sinani, and S. Sremac, "Golden ratio (sectiona aurea) in Markovian ants AI hybrid," in Adaptive and Intelligent Systems, vol. 6943 of Lecture Notes in Computer Science, pp. 356-367, Springer, New York, NY, USA, 2011.

[31] T. von Karman, "Progress in the statistical theory of turbulence," Proceedings of the National Academy of Sciences of the United States of America, vol. 34, no. 11, pp. 530-539, 1948.

[32] G. H. Goedecke, V. E. Ostashev, D. K. Wilson, and H. J. Auvermann, "Quasi-wavelet model of von Kármán spectrum of turbulent velocity fluctuations," Boundary-Layer Meteorology, vol. 112, no. 1, pp. 33-56, 2004.

[33] E. E. Morfiadakis, G. L. Glinou, and M. J. Koulouvari, “The suitability of the von Karman spectrum for the structure of turbulence in a complex terrain wind farm," Journal of Wind Engineering and Industrial Aerodynamics, vol. 62, no. 2-3, pp. 237-257, 1996.

[34] M. C. H. Hui, A. Larsen, and H. F. Xiang, "Wind turbulence characteristics study at the Stonecutters Bridge site: part II: wind power spectra, integral length scales and coherences," Journal of Wind Engineering and Industrial Aerodynamics, vol. 97, no. 1, pp. 48-59, 2009.

[35] G. Huang and X. Chen, "Wavelets-based estimation of multivariate evolutionary spectra and its application to nonstationary downburst winds," Engineering Structures, vol. 31, no. 4, pp. 976989, 2009.

[36] D. K. Wilson, V. E. Ostashev, and G. H. Goedecke, "Quasiwavelet formulations of turbulence and other random fields with correlated properties," Probabilistic Engineering Mechanics, vol. 24, no. 3, pp. 343-357, 2009. 
[37] G. Li and Q. Li, Theory of Time-Varying Reliability for Engineering Structures and Its Applications, Science Press, Beijing, China, 2001, (Chinese).

[38] J. Pang, Z. Lin, and Y. Lu, "Discussion on the simulation of atmospheric boundary layer with spires and roughness elements in wind tunnels," Experiments and Measurements in Fluid Mechanics, vol. 18, no. 2, pp. 32-37, 2004 (Chinese).

[39] Y. Q. Xiao, J. C. Sun, and Q. Li, “Turbulence integral scale and fluctuation wind speed spectrum of typhoon: an analysis based on field measurements," Journal of Natural Disasters, vol. 15, no. 5, pp. 45-53, 2006 (Chinese).

[40] J. C. Kaimal, J. C. Wyngaard, Y. Yzumi, and O. R. Cote, "Spectral characteristics of surface layer turbulence," Quarterly Journal of the Royal Meteorological Society, vol. 98, no. 417, pp. 563-589, 1972.

[41] H. A. Panofsky, D. Larko, R. Lipschutz et al., "Spectra of velocity components over complex terain," Quarterly Journal, Royal Meteorological Society, vol. 108, no. 455, pp. 215-230, 1982.

[42] M. Bauer and A. Zeibig, "Towards the applicability of the modified von Kármán spectrum to predict trailing edge noise," Notes on Numerical Fluid Mechanics, vol. 92, pp. 381-388, 2006.

[43] C. C. Tropea, A. L. Yarin, and J. F. Foss, Eds., Springer Handbook of Experimental Fluid Mechanics, Springer, New York, NY, USA, 2007.

[44] A. S. Monin and A. M. Yaglom, Statistical Fluid Mechanics: Mechanics of Turbulence, vol. 2, The MIT Press, Cambridge, Mass, USA, 1971, edited by J. L. Lumley.

[45] H. M. Hastings and G. Sugihara, Fractals, a User's Guide for the Natural Sciences, Oxford University Press, Oxford, UK, 1993.

[46] M. J. Jamieson, "Fibonacci numbers and Aitken sequences revisited," The American Mathematical Monthly, vol. 97, no. 9, pp. 829-831, 1990.

[47] D. L. Ranum, "On some applications of Fibonacci numbers," The American Mathematical Monthly, vol. 102, no. 7, pp. 640-645, 1995.

[48] M. H. Eggar, "Applications of Fibonacci numbers," The Mathematical Gazette, vol. 63, no. 423, pp. 36-39, 1979.

[49] B. N. N. Achar, J. W. Hanneken, and T. Clarke, "Damping characteristics of a fractional oscillator," Physica A, vol. 339, no. 3-4, pp. 311-319, 2004.

[50] B. N. N. Achar, J. W. Hanneken, and T. Clarke, "Response characteristics of a fractional oscillator," Physica A, vol. 309, no. 3-4, pp. 275-288, 2002.

[51] S. C. Lim and S. V. Muniandy, "Self-similar Gaussian processes for modeling anomalous diffusion," Physical Review E, vol. 66, no. 2, Article ID 021114, 14 pages, 2002.

[52] S. C. Lim and L. P. Teo, "The fractional oscillator process with two indices," Journal of Physics A, vol. 42, no. 6, Article ID 065208, 34 pages, 2009.

[53] C. H. Eab and S. C. Lim, "Fractional generalized Langevin equation approach to single-file diffusion," Physica A, vol. 389, no. 13, pp. 2510-2521, 2010.

[54] C. H. Eab and S. C. Lim, "Path integral representation of fractional harmonic oscillator," Physica A, vol. 371, no. 2, pp. 303316, 2006.

[55] S. C. Lim, M. Li, and L. P. Teo, "Langevin equation with two fractional orders," Physics Letters A, vol. 372, no. 42, pp. 63096320, 2008.

[56] J. Klafter, S. C. Lim, and R. Metzler, Fractional Dynamics: Recent Advances, World Scientific Publishing, Singapore, 2012.
[57] J. A. T. Machado, M. F. Silva, R. S. Barbosa et al., "Some applications of fractional calculus in engineering," Mathematical Problems in Engineering, vol. 2010, Article ID 639801, 34 pages, 2010.

[58] C. Cattani, "Fractional calculus and Shannon wavelet," Mathematical Problems in Engineering, vol. 2012, Article ID 502812, 26 pages, 2012.

[59] S. C. Lim and S. V. Muniandy, "Generalized Ornstein-Uhlenbeck processes and associated self-similar processes," Journal of Physics A, vol. 36, no. 14, pp. 3961-3982, 2003.

[60] W. T. Coffey, Y. P. Kalmykov, and J. T. Waldron, The Langevin Equation, vol. 14 of World Scientific Series in Contemporary Chemical Physics, World Scientific Publishing, Singapore, 2nd edition, 2004.

[61] J. S. Bendat and A. G. Piersol, Random Data: Analysis and Measurement Procedure, Wiley Series in Probability and Statistics, John Wiley \& Sons, New York, NY, USA, 3rd edition, 2000.

[62] C. M. Harris, Shock and Vibration Handbook, McGraw-Hill, New York, NY, USA, 4th edition, 1995.

[63] S. V. Gupta, Measurement Uncertainties, Springer, New York, NY, USA, 2012.

[64] W. Gautschi, Numerical Analysis, Springer, New York, NY, USA, 2nd edition, 2012.

[65] S. K. Mitra and J. F. Kaiser, Handbook for Digital Signal Processing, John Wiley \& Sons, New York, NY, USA, 1993.

[66] M. Drosg, Dealing with Uncertainties: A Guide to Error Analysis, Springer, New York, NY, USA, 2nd edition, 2009.

[67] I. M. Gelfand and K. Vilenkin, Generalized Functions, vol. 1, Academic Press, New York, NY, USA, 1964.

[68] M. Li and S. C. Lim, "A rigorous derivation of power spectrum of fractional Gaussian noise," Fluctuation and Noise Letters, vol. 6, no. 4, pp. C33-C36, 2006.

[69] P. Hall and R. Roy, "On the relationship between fractal dimension and fractal index for stationary stochastic processes," The Annals of Applied Probability, vol. 4, no. 1, pp. 241-253, 1994.

[70] B. B. Mandelbrot, Gaussian Self-Affinity and Fractals, Springer, New York, NY, USA, 2001.

[71] C. Cattani, G. Pierro, and G. Altieri, "Entropy and multifractality for the myeloma multiple TET 2 gene," Mathematical Problems in Engineering, vol. 2012, Article ID 193761, 14 pages, 2012.

[72] E. G. Bakhoum and M. H. M. Cheng, "Miniature carbon monoxide detector based on nanotechnology," IEEE Transactions on Instrumentation and Measurement, vol. 62, no. 1, pp. 240-245, 2013.

[73] E. G. Bakhoum, "High-sensitivity miniature smoke detector," IEEE Sensors Journal, vol. 12, no. 10, pp. 3031-3035, 2012.

[74] C. Toma, "Advanced signal processing and command synthesis for memory-limited complex systems," Mathematical Problems in Engineering, vol. 2012, Article ID 927821, 13 pages, 2012.

[75] E. G. Bakhoum and C. Toma, "Modeling transitions in complex systems by multiplicative effect of temporal patterns extracted from signal flows," Mathematical Problems in Engineering, vol. 2012, Article ID 409856, 11 pages, 2012.

[76] M. Pinchas, "Symbol error rate as a function of the residual ISI obtained by blind adaptive equalizers for the SIMO and fractional Gaussian noise case," Mathematical Problems in Engineering, vol. 2013, Article ID 860389, 9 pages, 2013.

[77] L. X. Li, C. Yang, S. L. Hui et al., "A reconfigurable logic cell based on a simple dynamical system," Mathematical Problems in Engineering. In press. 
[78] Y. M. Wang, "Maximum-norm error estimates of ADI methods for a two-dimensional fractional sub-diffusion equation," Advances in Mathematical Physics. In press.

[79] Z. Wang and L. T. Yan, "The S-transform of sub-fBm and an application to a class of linear sub-fractional BSDEs," Advances in Mathematical Physics, vol. 2013, Article ID 827192, 11 pages, 2013.

[80] H. P. Peng, G. Hu, L. X. Li, Y. X. Yang, and J. H. Xiao, "Constructing dynamic multiple-input multiple-output logic gates," Mathematical Problems in Engineering, vol. 2011, Article ID 380345, 12 pages, 2011.

[81] H. P. Peng, L. X. Li, Y. X. Yang, and F. Sun, "Conditions of parameter identification from time series," Physical Review E, vol. 83, no. 3, Article ID 036202, 8 pages, 2011.

[82] L. X. Li, J. H. Xiao, H. P. Peng, Y. X. Yang, and Y. H. Chen, "Improving synchronous ability between complex networks," Nonlinear Dynamics, vol. 69, no. 3, pp. 1105-1110, 2012. 


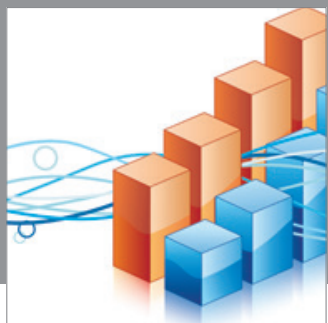

Advances in

Operations Research

mansans

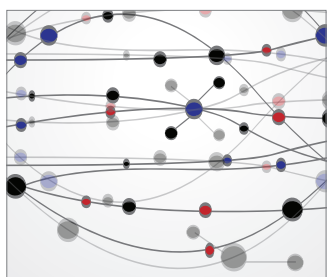

The Scientific World Journal
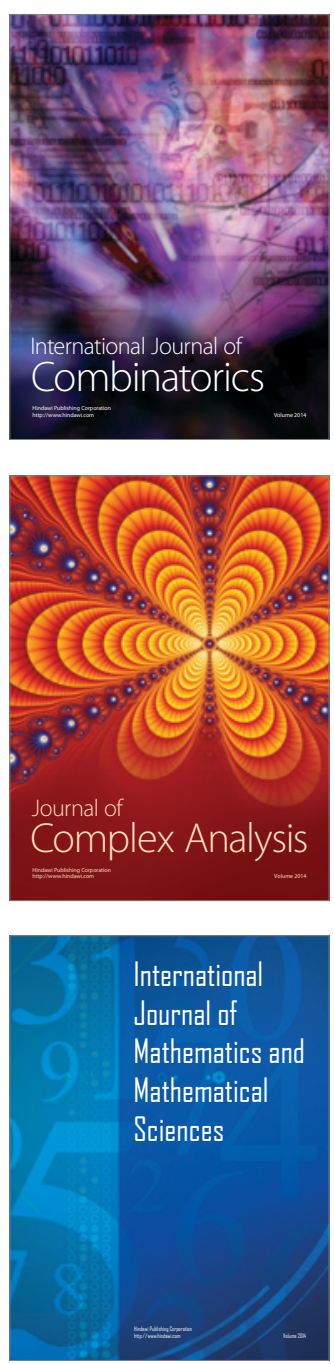
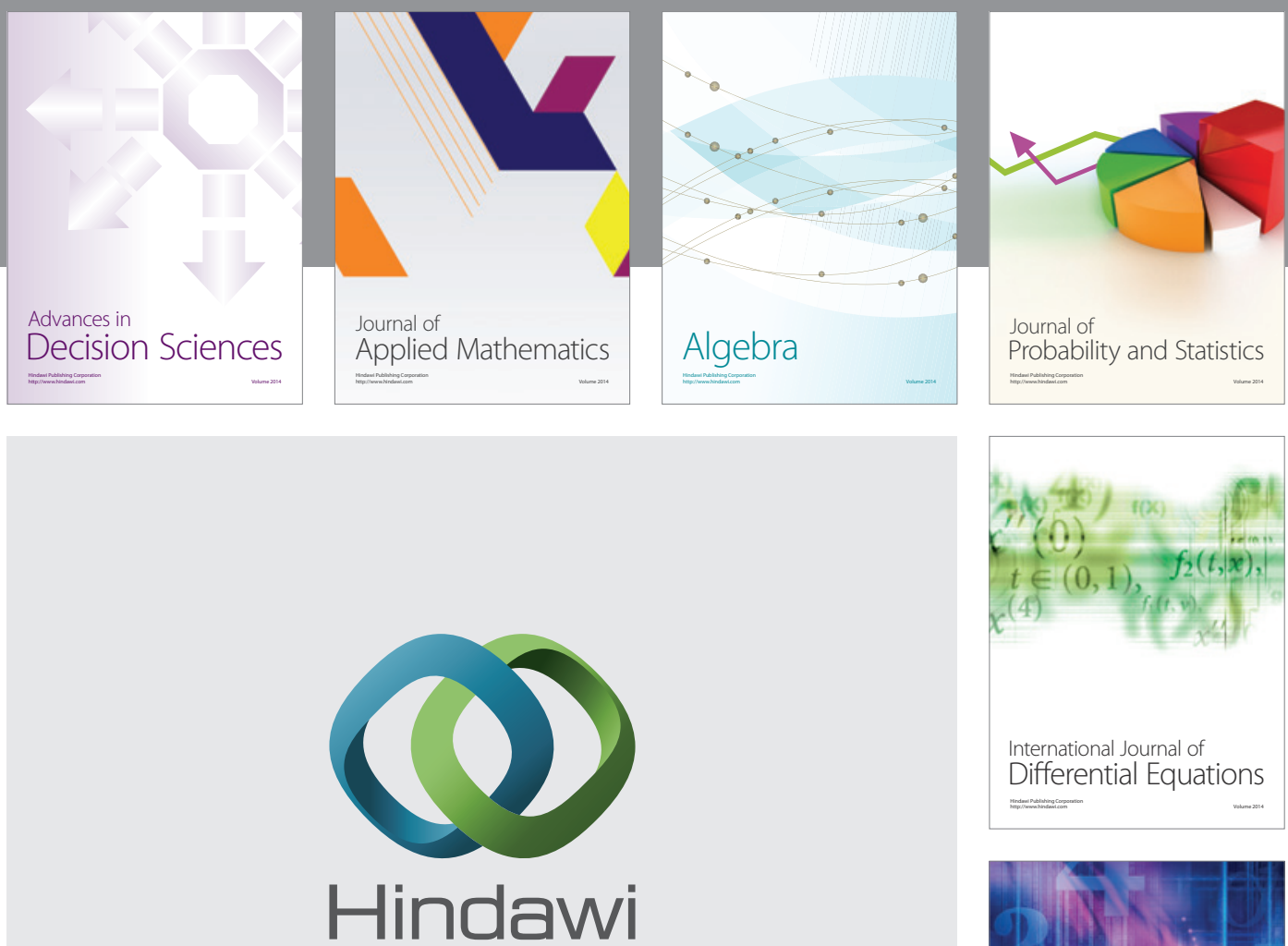

Submit your manuscripts at http://www.hindawi.com
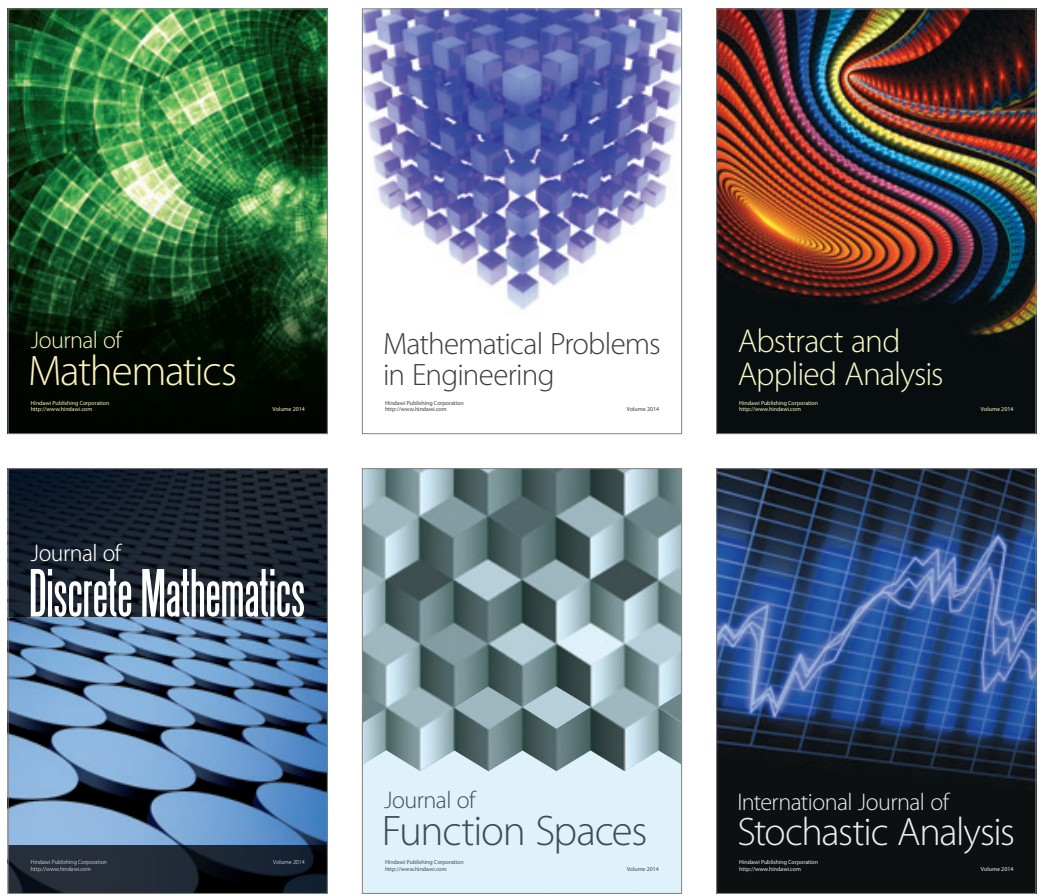

Journal of

Function Spaces

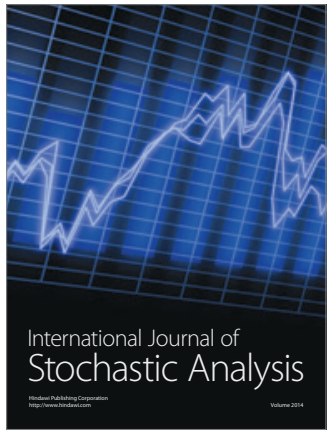

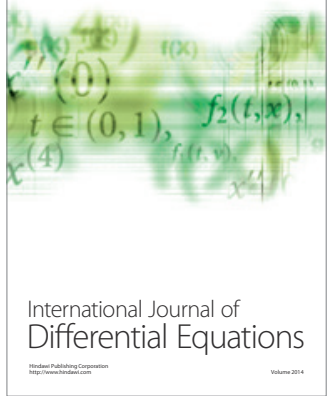
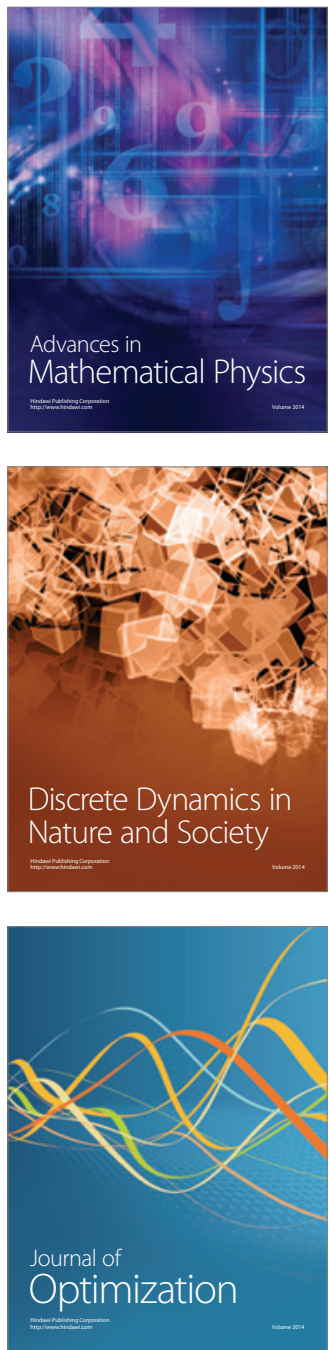